\author{
Amir H. Behbahani ${ }^{1}$ \\ Department of Mechanical and \\ Aerospace Engineering, \\ University of California, Los Angeles, \\ Los Angeles, CA 90095 \\ Robert T. M'Closkey² \\ Professor \\ Department of Mechanical and \\ Aerospace Engineering, \\ University of California, Los Angeles, \\ Los Angeles, CA 90095 \\ e-mail: rtm@seas.ucla.edu
}

\section{Multimodal Tuning of an Axisymmetric Resonator}

This paper reports an approach for the simultaneous elimination of the modal frequency differences within two pairs of modes in an axisymmetric resonator. Fabricated devices exhibit frequency detuning, which can be eliminated by strategically mass loading the resonator. Each pair of modes responds to the mass loading differently so models are developed to predict the postloading frequency differences. The models are incorporated into a search procedure to select deposition sites that simultaneously reduce the modal frequency difference within each pair. The proposed approach is successfully implemented on a resonator whose modal frequency differences are reduced below $200 \mathrm{mHz}$ from an initial frequency difference of $23.5 \mathrm{~Hz}$ for a pair of modes at $13.8 \mathrm{kHz}$, and a $2.4 \mathrm{~Hz}$ difference for another pair of modes at $24.3 \mathrm{kHz}$. [DOI: 10.1115/1.4043331]

\section{Introduction}

Microscale axisymmetric resonators are the foundation of a new class of planar high-performance Coriolis vibratory gyros (CVGs) [1-4]. The axisymmetry imparts the nominal degeneracy of one or more pairs of modal frequencies in the resonator. Modal degeneracy maximizes the signal-to-noise ratio (SNR) of the angular rate measurement relative to noise introduced by electronic buffers that amplify the small currents present at the sensing electrodes so any departure from "tuned" modal frequencies will degrade the SNR especially in resonators with high quality factors [5]. Although the resonators are fabricated using etch processes that enable a high degree of dimensional precision, asymmetries are inevitably introduced, which detunes the pairs of modal frequencies. The detuning must be eliminated in order to recover the highest achievable SNR. A ubiquitous method, which will not be reviewed here, is to electrically bias electrodes relative to the resonator in order to create electrostatic "springs." One potential disadvantage of this approach is the requirement it places on the stability of the electrical biases because any drift in the biases will result in small perturbations to the resonator dynamics. Alternatively, permanent modification of the resonator can be achieved by selectively adding or removing mass in order to modify the local stiffness and/or mass distribution of the resonator.

This paper reports the simultaneous elimination of the modal frequency differences within two pairs of modes near $14 \mathrm{kHz}$ and $24 \mathrm{kHz}$ in a planar axisymmetric resonator using a permanent mass deposition approach. The motivation for pursuing tuning of two pairs of modes within the same resonator was demonstrated in Ref. [6] where it was shown that each pair of modes can function as a CVG thereby yielding two angular rate measurements from a single resonator. The zero-rate offset drifts were highly correlated over long time intervals and so a technique was proposed to fuse the two measurements into a single angular rate estimate with a much more stable offset. The resonator in Ref. [6] is also used in this paper; however, in Ref. [6], it was modified using an ad hoc approach of essentially tuning one pair first, and then attempting to tune the second pair without disturbing the modal frequency difference of the first pair. This largely trial-and-error approach did eventually tune both pairs of modal frequencies; however, this paper presents and demonstrates a more systematic approach for achieving the same tuning goal despite the significant challenges posed by the fact the physical perturbations are

${ }^{1}$ Present address: Biology and Biological Engineering, California Institute of Technology, Pasadena, CA.

${ }^{2}$ Corresponding author.

Contributed by the Dynamic Systems Division of ASME for publication in the Journal of Dynamic Systems, Measurement, and Control. Manuscript received June 19, 2018; final manuscript received March 19, 2019; published online May 2, 2019. Assoc. Editor: Douglas Bristow. quantized in both mass and location. Although it is generally not possible to achieve complete frequency degeneracy within each pair, it was shown in Ref. [5] that some small residual detuning can be tolerated in CVG applications. Other contributions of this paper include a technique for identifying the modal frequencies and mode shapes from transient response data. This is a complementary technique to the ubiquitous use of empirical frequency response estimates produced by a signal analyzer.

Relatively little research on the permanent tuning of axisymmetric microscale has been reported in the literature. In addition to the experiments with the same resonator design reported in Refs. [7] and [8], gold was ablated from the lip of a microscale hemispherical resonator in Ref. [9] and laser cutting of a silicon ring was reported in Ref. [10]. Modification of the so-called wine glass modes was the objective in these studies. Despite the lack of results specific to microscale resonators, there is a large body of literature concerning the tuning of rings, bells, and cylinders, for example see Refs. [11-13]. Of particular relevance are the analytical results of Refs. [14] and [15], which focus on the tuning of slightly imperfect rings. The results from these papers are adapted here to develop models, which relate the pre- and post-perturbation modal frequency differences and also address the multiring structure of the resonator under consideration in this paper. The solution proposed in Ref. [15] to simultaneously tune multiple pairs of modes within an imperfect ring cannot be applied here, though, because the mass deposition locations and mass sizes are quantized. Thus, this paper employs a search procedure to identify deposition locations, which will reduce the modal frequency differences below a specified threshold.

The paper is organized as follows: Section 2 discusses a technique for estimating the tangential and radial velocity components at the potential deposition sites. These components are needed in order to develop the modal frequency perturbation model. Section 3 describes how the resonator is modeled from transient response data. The resonator models yield estimates of the modal frequencies and the corresponding mode shapes for each pair of modes. The large number of spatially distributed pick-offs provides accurate measurements of the mode shapes. Section 4 develops the modal frequency perturbation model based on small point mass perturbations of a simple ring. The model is generalized to the resonator under study using the velocity component analysis from Sec. 2. Section 5 proposes an optimization problem to search for suitable deposition locations, given constraints on the maximum number of solder spheres that can be used in a given iteration. This is a global optimization problem because of the quantization of the deposited mass as well as the quantized deposition locations. Section 6 demonstrates the tuning process on a resonator with initial modal frequency differences of $23.5 \mathrm{~Hz}$ and $2.4 \mathrm{~Hz}$ within different pairs of modes. The intermediate deposition steps and frequency response measurements of the resonator are also 
provided. Three iterations are required to reduce the model frequency differences below $200 \mathrm{mHz}$. Section 7 concludes the paper.

\section{Resonator Modeling}

The planar resonator used in this study is shown in Fig. 1. The resonator consists of nine concentric rings, each of which is connected to its neighbor(s) by a staggered system of spokes. The outer diameter of the resonator is $1 \mathrm{~cm}$ and each ring is approximately $20 \mu \mathrm{m}$ wide. The resonator thickness is $270 \mu \mathrm{m}$. The mass distribution of the resonator is modified in order to reduce the modal frequency difference within selected pairs of modes and the four outermost layers of spokes are larger $(130 \mu \mathrm{m}$ in diameter) and are targets for mass deposition. This essential resonator design is also employed in Refs. [7] and [8]; however, there are differences in the way mass perturbations are achieved and, consequently, there are small changes to the top surface of the resonator to support one particular method or another. In this paper, a $1 \mu \mathrm{m}$ thick, $130 \mu \mathrm{m}$ diameter, gold film is deposited on the top surface of all 96 "large" spokes. These are potential targets for mass deposition by reflowing solder spheres of $75 \mu \mathrm{m}$ and $35 \mu \mathrm{m}$ diameters-see Fig. 2. In Ref. [7], the outermost layer of spokes had shallow reservoirs into which silver particle laden ink was deposited. This reference lacked the smaller diameter solder spheres so the ink was necessary for fine tuning. More recently, [8] demonstrated selective etching of the top surface of the large spokes, i.e., mass is removed.

The resonator is instrumented with eight pick-offs that measure the radial velocity of the outer-most ring. These pick-offs are denoted $S_{1}-S_{8}$ in Fig. 1. One advantage over the authors' prior work is the much greater visibility into the mode shapes of the resonator because of the use of eight distinct pick-offs. The resonator is forced by applying potentials, relative to the resonator's constant potential, to the $D_{1}, D_{6}$, and $D_{8}$ electrodes also shown in this figure. A broadband frequency response from electrode $D_{1}$ to pick-off $S_{7}$ is shown in Fig. 3 and reveals modes near 6.5, 14 and $24 \mathrm{kHz}$. Measurements in Sec. 3 demonstrate that there are in fact pairs of modes at these frequencies-individual modes within a given pair cannot be distinguished on this scale. The pairs are identified according to the integer $n$ with the pair near $6.5 \mathrm{kHz}$ designated the $n=1$ pair, the pair near $14 \mathrm{kHz}$ designated the

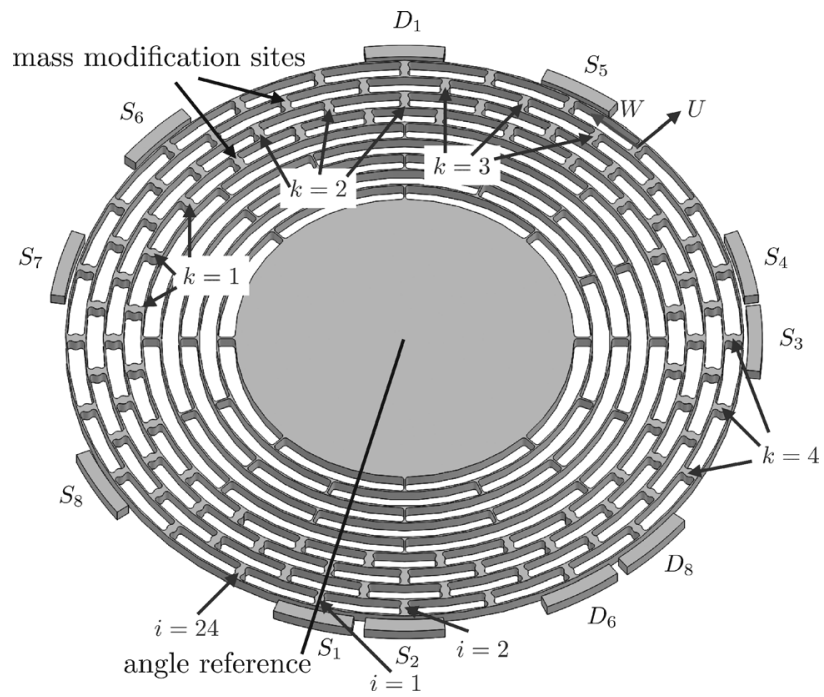

Fig. 1 Isometric view of the $1 \mathrm{~cm}$ diameter resonator. The large spoke positions are identified with the indices $i \in\{1,2, \ldots, 24\}$ denoting the angular position, and $k \in\{1,2,3,4\}$ denoting the radial position. The electrodes labeled $D_{1}, D_{6}$, and $D_{8}$ are used for exciting the resonator and the electrodes labeled $S_{1}-S_{8}$ are used for measuring the radial velocities at points along the outermost ring.

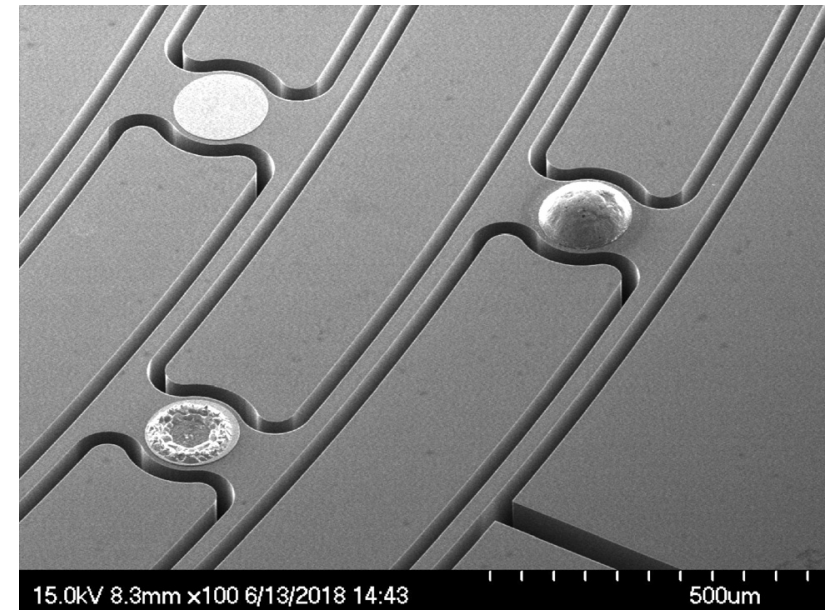

Fig. 2 A view of reflowed solder spheres in the area between the $S_{6}$ and $D_{1}$ electrodes. A $75 \mu \mathrm{m}$ diameter solder sphere is on the upper right spoke and a reflowed $35 \mu \mathrm{m}$ diameter solder sphere is on bottom left spoke.

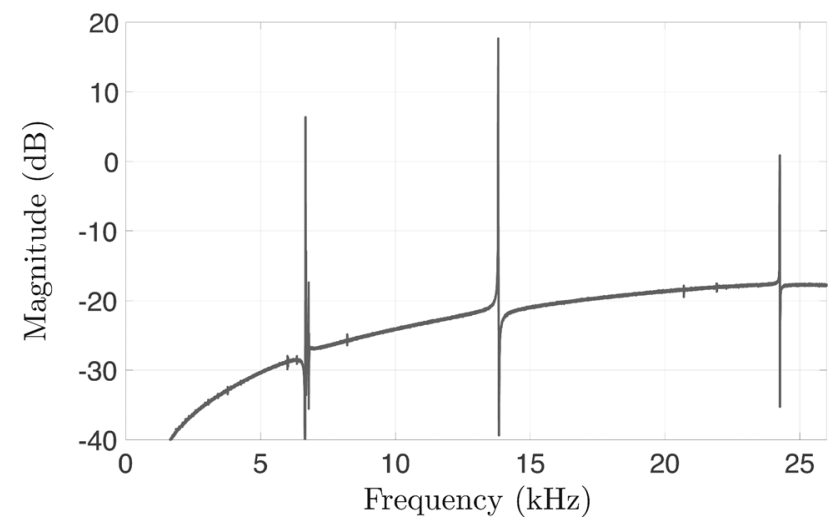

Fig. 3 Broadband frequency response using electrode $D_{1}$ for excitation and electrode $S_{7}$ for sensing. The $n=1,2,3$ pairs of modes are located near 6,14 , and $24 \mathrm{kHz}$, respectively.

$n=2$ pair, and so forth. The labeling convention derives from the dominant $n \theta$ dependence of the radial and tangential displacements associated with the mode shapes, where $\theta$ is the angular coordinate measured relative to the reference shown in Fig. 1.

The perturbation models discussed in Sec. 4 require estimates of the relative amplitudes of the radial and tangential velocities at the large spokes, denoted $U$ and $W$, respectively. Thus, it is necessary to enumerate these locations according to the "layer" (radial location) and position within the layer (angular location). This is accomplished by using index $k \in\{1,2,3,4\}$ to denote the layer (with $k=1$ being the innermost layer of large spokes) and index $i$ $\in\{1,2, \ldots, 24\}$ to denote the angular position relative to a datum defined as the radial line that bisects the electrode labeled $S_{1}$ in Fig. 1. The angular location of a given spoke is then denoted $\theta_{k, i}$. The spoke velocities are estimated by applying finite element analysis to multiple cases of slightly perturbed resonators. Each case study produces mode shapes with different spatial orientation; however, they are aggregated into estimates of amplitudes of radial and tangential velocity components by computing the discrete Fourier series of the 24 radial spoke velocities (similarly, the tangential velocities) for each spoke layer. Although the phases of the Fourier components differ from one case to the other, the amplitude ratios are essentially constant and independent of the perturbation magnitude as shown in Ref. [8] where only the $n=2$ pair of modes is analyzed. An example of the mode shapes associated with the $n=2,3$ pairs of modes is shown in Fig. 4 . 


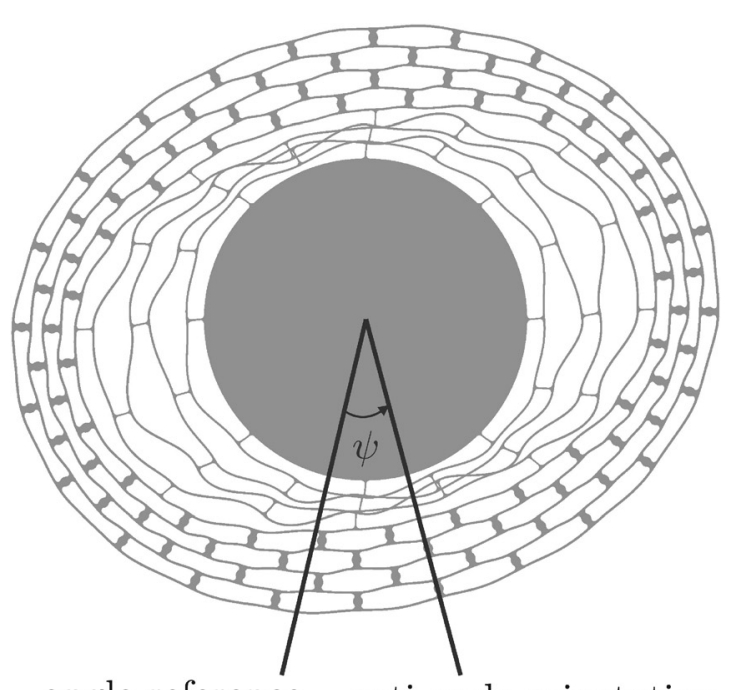

angle reference anti-node orientation

(a)

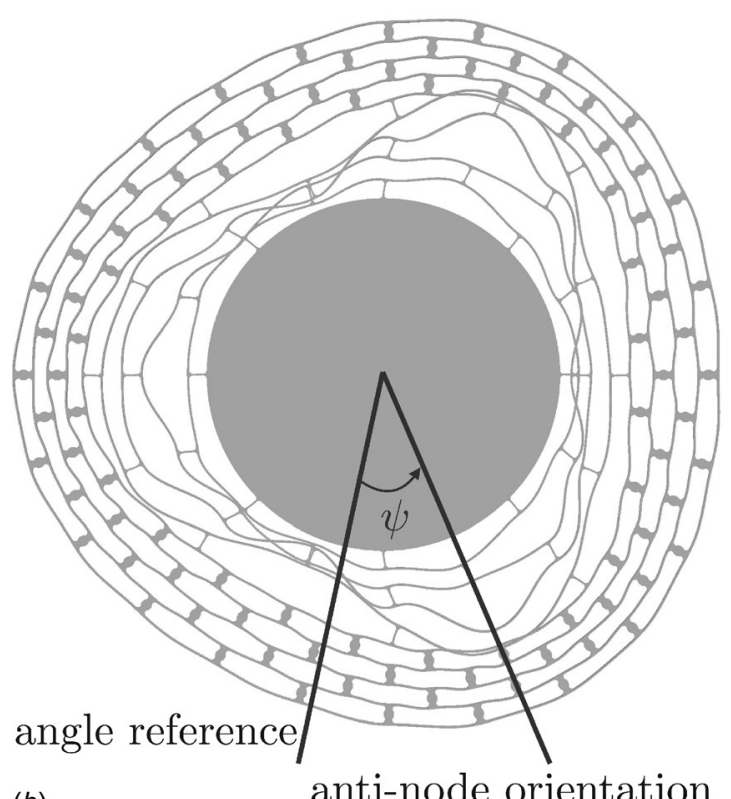

(b)

anti-node orientation

Fig. 4 Mode shapes for the $n=2,3$ modes. Only one mode in each pair is shown.

The radial and tangential amplitude ratios are graphed in Figs. 5 and 6 for $n=2$ and $n=3$, respectively. In the $n$th pair of modes, the dominant harmonic of the mode shape is the $n \theta$ harmonic. Figure 5 also indicates that the $6 \theta$ and $10 \theta$ harmonics have the next largest amplitudes for the radial components but only the $2 \theta$ harmonic is significant for the tangential motion. For the $n=3$ pair of modes, the $3 \theta$ harmonics are dominant in the mode shapes as shown in Fig. 6; however, the $5 \theta$ and $11 \theta$ harmonics are also present in the $n=3$ mode shapes for the radial component. Since amplitude ratios are shown in these figures, the normalization is performed with respect to the largest harmonic amplitude for each $n$, which is the $2 \theta$ radial velocity harmonic corresponding to the innermost layer of spokes $(k=1)$ when $n=2$, and the $3 \theta$ radial velocity harmonic corresponding to the innermost layer of spokes when $n=3$. For the dominant harmonic terms only, the radial amplitude ratios are denoted by $\alpha_{n, k}$ and the tangential amplitude ratios are denoted by $\beta_{n, k}$. Based on the normalization discuss above, $\alpha_{2,1}=1$ and $\alpha_{3,1}=1$. A numerical summary of the ratios shown in Figs. 5 and 6 is given in Table 1 for $n=2,3$.

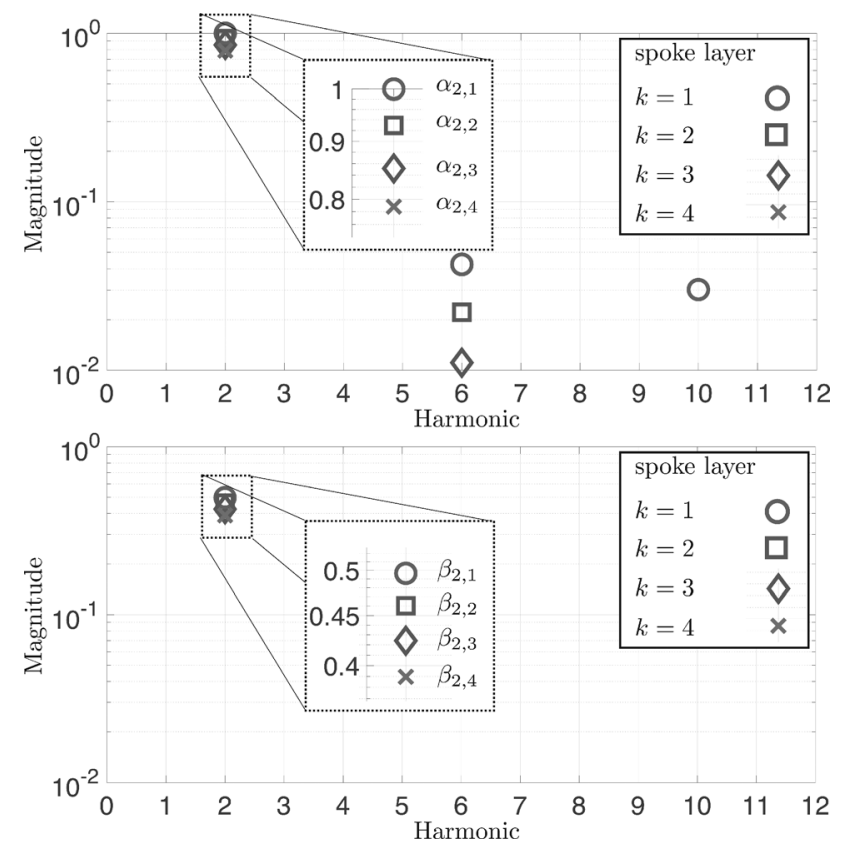

Fig. 5 (Top) The normalized magnitudes of the Fourier series coefficients for the spoke radial velocities at different layers for the $n=2$ modes. (Bottom) The normalized magnitudes of the Fourier series coefficients for the spoke tangential velocities at different layers for the $n=2$ modes.
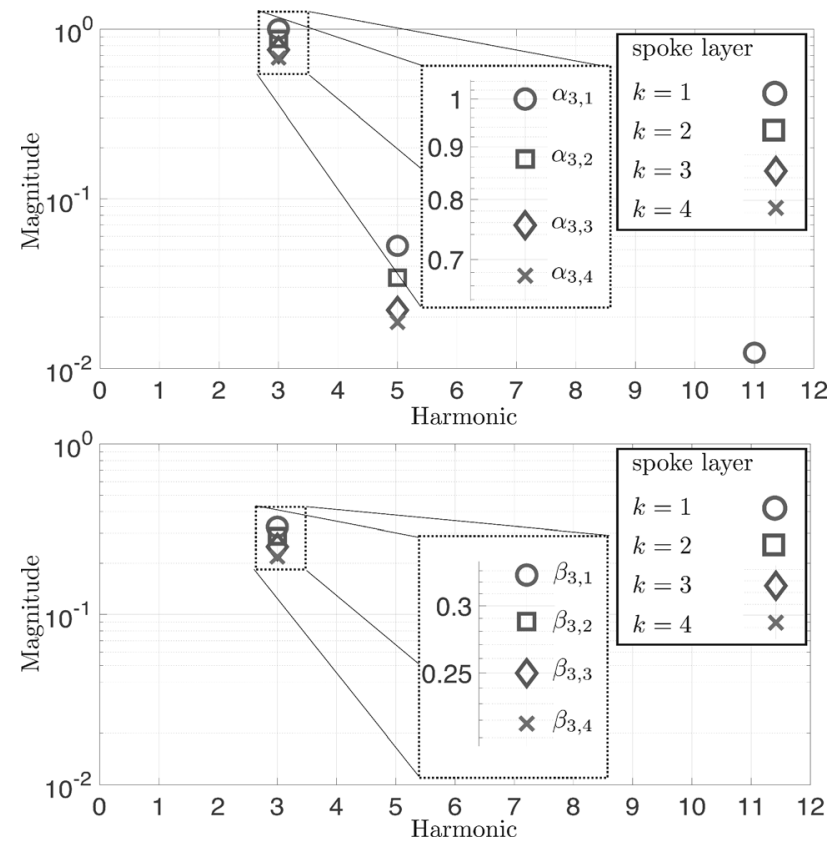

Fig. 6 (Top) The normalized magnitudes of the Fourier series coefficients for the spoke radial velocities at different layers for the $n=3$ modes. (Bottom) The normalized magnitude of the Fourier series coefficients for the spoke tangential velocities at different layers for $n=3$ modes.

The antinodes of the $n$th pair of mode are defined as the angle where the radial velocity components achieve their maximum value for the dominant $n \theta$ harmonics. The finite element analysis also reveals that the antinodes are equal across spoke layers. In other words, the antinodes are not a function of $k$ when considering the $n \theta$ harmonics. The orientation of the $n \theta$ harmonic in one of the $n$th modes is denoted by $\psi$. For example, the antinode 
Table 1 Normalized amplitudes of spoke velocity components

\begin{tabular}{llllll}
\hline \hline & \multicolumn{2}{c}{$n=2$} & & \multicolumn{2}{c}{$n=3$} \\
\cline { 2 - 3 } \cline { 5 - 6 } Layer, $k$ & $\alpha_{2, k}$ & $\beta_{2, k}$ & & $\alpha_{3, k}$ & $\beta_{3, k}$ \\
\hline 1 & 1 & 0.4969 & 1 & 0.3257 \\
2 & 0.9289 & 0.4603 & & 0.8741 & 0.2878 \\
3 & 0.8506 & 0.4214 & 0.7543 & 0.2492 \\
4 & 0.7868 & 0.3886 & 0.6750 & 0.2171 \\
\hline \hline
\end{tabular}

associated with the mode shape of one mode within each $n=2,3$ pair is shown in Fig. 4.

The practical problem of measuring the phases (angular orientation) of the spatial harmonics also poses challenges - the peripheral electrodes illustrated in Fig. 1 produce signals proportional to the radial velocities at points on the outermost ring, but this is where all harmonics have their smallest relative amplitudes. For example, the $6 \theta$ harmonic for the $n=2$ radial velocity at the outermost ring is about one-hundredth of the $2 \theta$ harmonic. The $10 \theta$ harmonic is even smaller. It will be shown in Sec. 3 that it is not possible to measure the $6 \theta$ and $10 \theta$ harmonics associated with the $n=2$ mode-shapes. A similar conclusion is reached concerning the $n=3$ radial velocity, i.e., the $5 \theta$ and $11 \theta$ harmonics cannot be measured. Thus, only the $2 \theta$ harmonics for the $n=2$ pair, and the $3 \theta$ harmonics for the $n=3$ can be reliably measured with the present electrode arrangement. Since it is not possible to infer the phases associated with the higher harmonics in a given $n=2,3$ mode, and owing to smaller contribution of these harmonics to the spoke velocity, the radial and tangential velocities in a given spoke layer are approximated by retaining only the dominant harmonic, i.e., $2 \theta$ harmonic for $n=2$ and $3 \theta$ harmonic for $n=3$. Thus, the radial and tangential velocities of the spoke in the $k$ th layer and at the $i$ th angular position for an $n=2$ or $n=3$ mode are proportional to

$$
\begin{aligned}
& U(\theta) \propto \alpha_{n, k} \cos \left(n\left(\theta_{k, i}-\psi\right)\right) \\
& W(\theta) \propto \beta_{n, k} \sin \left(n\left(\theta_{k, i}-\psi\right)\right)
\end{aligned}
$$

The angle $\theta_{k, i}$ denotes the angular position of the spoke relative to the datum, and $\psi$ is the antinode orientation of the $n \theta$ harmonic. Note that Table 1 shows $\alpha_{n, k} / \beta_{n, k} \approx n$, that is, the ratio of the radial velocity amplitude to the tangential velocity amplitude in the $k$ th spoke layer is essentially equal to $n$ for the $n$th pair of modes. This relation is also satisfied by a uniform inextensible ring because $U=\partial W / \partial \theta$ in this case [16]. This also explains why $U$ and $W$ are in quadrature in Eq. (1).

\section{Modal Parameter Measurements}

Modeling the resonator dynamics from measurement data is discussed in this section. The perturbation models in Sec. 4 require measurements of the difference between the modal frequencies within a given pair as well as estimates of the antinodes associated with the dominant harmonic of the mode shapes. The resonator's cofabricated electrodes are convenient for measuring the outer ring's radial velocity at well-defined points. It is assumed that the electrode signal is proportional to the outer ring's radial motion at the electrode's centroid. Prior research focused on fitting an "impedance" model to two-input/two-output frequency response measurements [7], but this technique did not take advantage of the higher spatial resolution provided by individual electrodes because three adjacent electrodes were combined into a "single" larger electrode for sensing the $n=2$ modes. The larger electrode area boosts the SNR but at the expense of lower spatial resolution; however, higher spatial resolution is necessary for accurately estimating the $n=2,3$ antinodes.

The resonator under consideration is also subject to parasitic coupling from the excitation electrodes to the pick-off electrodes, which can bias the frequency response estimates. These challenges motivated the search for other parametric modeling techniques that would yield not only information on the modal frequencies but also greater insight into the antinode orientations. The technique that is employed is based on models fit to transient response data. The general approach is described in Ref. [17] for a two-input/two-output resonator; however, for the present research, the technique was extended to include additional input-output channels. In addition to providing higher spatial resolution than previous efforts, the modeling technique has also been shown to reject any direct pick-off/forcer coupling due to the burst excitation nature of the inputs used for generating response data. For modeling the $n=2$ pair of modes, electrodes $D_{1}$ and $D_{8}$ in Fig. 1 are used for exciting the resonator and electrodes $S_{1}-S_{8}$ are used as pick-offs. The $n=3$ pair of modes is modeled from data obtained with the $D_{1}$ and $D_{6}$ excitation electrodes and, again, $S_{1}-S_{8}$ for the pick-offs. In this manner, a two-input/eight-output parametric model is derived for the $n=2$, and separately for the $n=3$, pair of modes. The models are discrete-time linear statespace models of the form

$$
\mathbf{x}^{+}=A \mathbf{x}+B \mathbf{u}, \quad \mathbf{s}=C \mathbf{x}
$$

where $A \in \mathbf{R}^{4 \times 4}, B \in \mathbf{R}^{8 \times 2}, C \in \mathbf{R}^{8 \times 4}$, $\mathbf{x}$ represents the state vector at the current time sample and $\mathbf{x}^{+}$is the state one sample in the future. The output vector is given by $\mathbf{s}=\left[\begin{array}{llll}s_{1}, & s_{2}, & \cdots & s_{8}\end{array}\right]^{\mathrm{T}}$, where $s_{k}$ represents the signal obtained from the electrode designated $S_{k}$ in Fig. 1. The input is $\mathbf{u}=\left[\begin{array}{ll}d_{1}, & d_{8}\end{array}\right]^{\mathrm{T}}$, respectively, $\mathbf{u}=\left[\begin{array}{ll}d_{1}, & d_{6}\end{array}\right]^{\mathrm{T}}$, for the $n=2$ pair, respectively, $n=3$ pair, where $d_{1}$ represents the stimulus applied to the $D_{1}$ electrode and so forth. The frequency responses of the identified modes are compared to the empirical frequency responses in Figs. 7 and 8. Only two input-output channels are shown for each pair of modes despite the fact that there are eight output channels and two input channels. These additional details are not shown for the sake of brevity. Note, however, that the parametric modeling technique rejects parasitic capacitive coupling and, thus, the model and empirical frequency responses deviate from each other away from the resonant frequencies. The parasitic coupling is especially evident in the $n=3$ empirical frequency response-the coupling has a tendency to create the flat floor in the frequency response magnitude. Note that these frequency responses represent the detail in a neighborhood of the $n=2,3$ modes shown in the broadband frequency response in Fig. 3. It is clear that the modes appear in pairs.

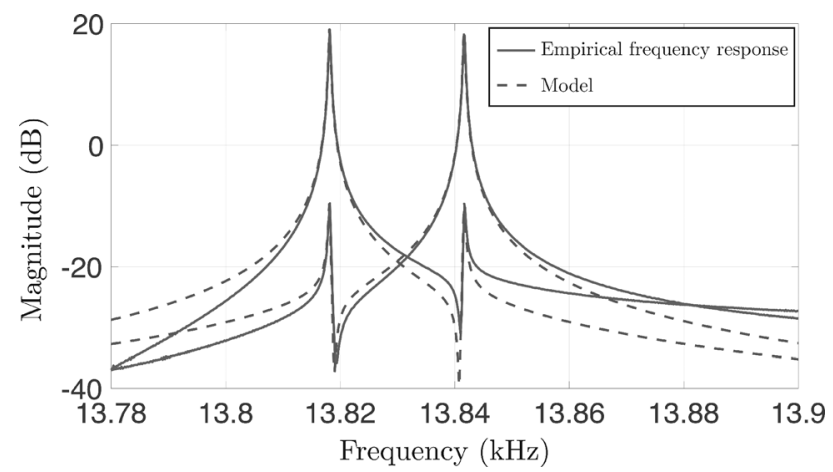

Fig. 7 Frequency response magnitude plot showing the $n=2$ modes prior to any mass deposition (the "as-fabricated" state of the resonator). The initial modal frequency difference is $23.49 \mathrm{~Hz}$. The frequency response of an identified parametric model is also shown. The deviation between the model and empirical data is due to parasitic coupling which is present in the empirical frequency response but not the parametric model. The $S_{2} / D_{1}$ and $S_{6} / D_{8}$ measurement channels are shown (see Fig. 1). 


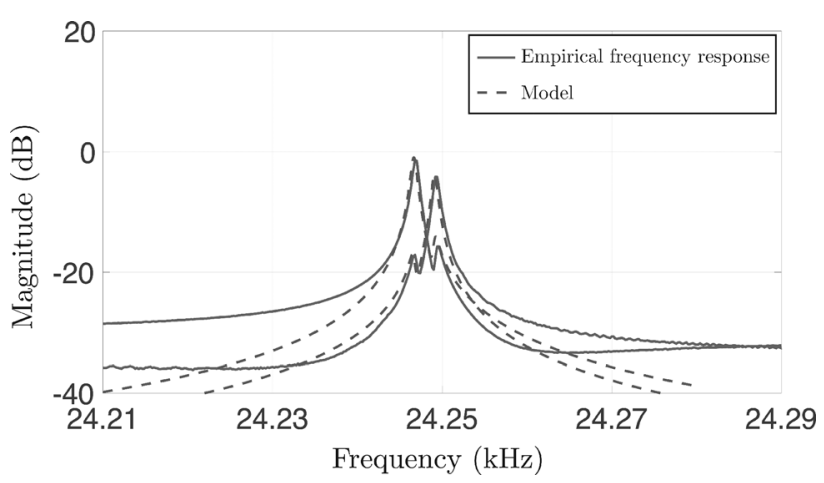

Fig. 8 Frequency response magnitude plot showing the $n=3$ modes prior to mass deposition. The initial modal frequency difference is $2.43 \mathrm{~Hz}$. The parasitic capacitive coupling creates the "floor" in the empirical frequency response. The $S_{2} / D_{1}$ and $S_{5} / D_{6}$ measurement channels are shown.

The modal frequencies and damping are determined from the eigenvalues of $A$ and the antinode orientations are estimated by analyzing its eigenvectors: let $\lambda$ represent an eigenvalue with corresponding eigenvector $\mathbf{v}$, then, the modal response corresponding to this eigenvalue as measured by the pick-offs is $\mathbf{s}\left(p t_{s}\right)$ $=C \mathbf{v} \lambda^{p}, p=0,1,2,3, \ldots$, where $t_{s}$ is the sample period associated with the model. The amplitude of the modal response in each output measurement channel can be determined by analyzing the elements of $C \mathbf{v}$. For example, Fig. 9 shows a graph of the elements of $C \mathbf{v}$ in the complex plane for a mode in the $n=2$ model. The fact that these elements are nearly colinear implies that measurement signals pass through zero at the same points in time as the modal response decays. Thus, removing the common phase term in each element allows us to graph the amplitude-with a signof the mode-shape at the corresponding location of the electrode. These elements are graphed in Figs. 10 and 11 for both modes in the $n=2$ and $n=3$ pairs. The angle $\theta=0$ corresponds to the location of the $S_{1}$ pick-off so its signed amplitude is graphed at $0 \mathrm{deg}$; the signed amplitude at the $S_{2}$ pick-off is graphed at $\theta=15 \mathrm{deg}$, and so forth. Also shown as a dashed line is a model of the form $\cos (n(\theta-\psi))$, which is determined from a least-squares fit, and where $\psi$ represents the antinode orientation associated with the mode. For the $n=2$ modes, $\psi_{2,1}$ represents the antinode orientation for the lower frequency mode and $\psi_{2,2}$ the antinode orientation for the higher frequency mode. The difference between the

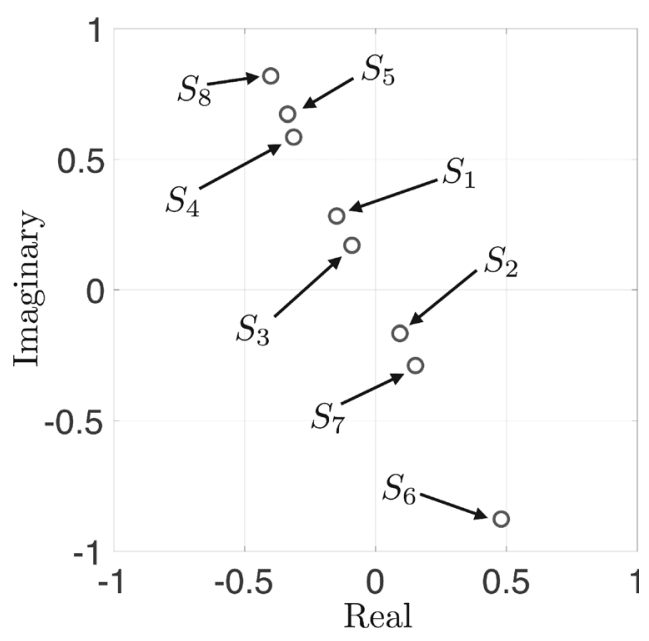

Fig. 9 Elements of $C v$ for an $n=2$ mode with labels indicating the associated electrode (see Eq. (2)). A shift in phase aligns these elements on the real axis-these are the values that a graphed in Figs. 10 and 11.

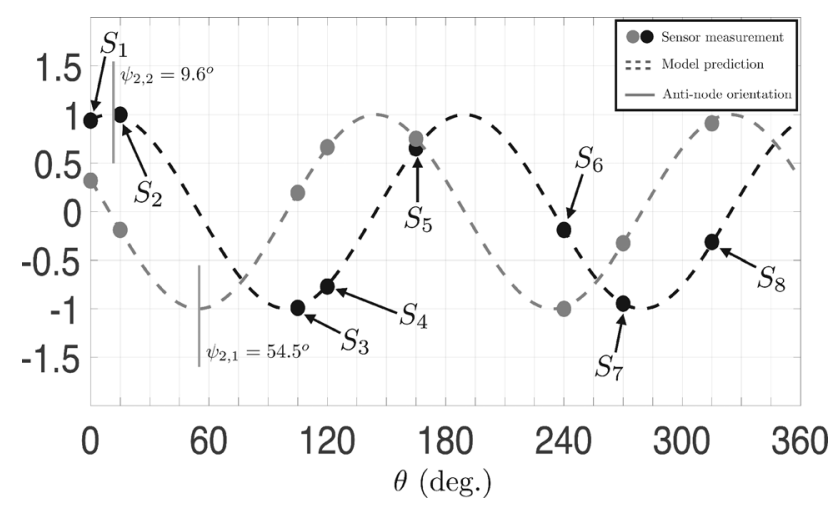

Fig. 10 Estimation of $n=2$ antinodes. The points are from the analysis of $C_{V}$ and the dashed line is the model $\cos (2(\theta-\psi))$ where $\psi$ is chosen to interpolate the points. Note that $\psi_{2,2}-\psi_{2,1}=-44.9$ deg.

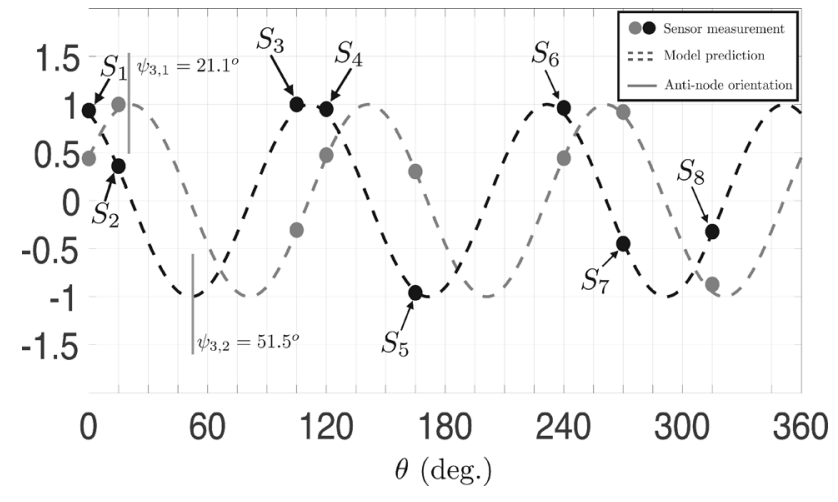

Fig. 11 Estimation of $n=3$ antinodes. Note that $\psi_{3,2}-\psi_{3,1}=30.4$ deg.

antinode orientations within a given pair of modes was not constrained to satisfy $\pm \pi /(2 n)$ rad yet it appears that the experiments confirm this relationship when $n=2,3$. This relation between antinode orientations is also satisfied by a single ring resonator [16]. Thus, the modeling technique provides accurate estimates of the modal frequencies and antinode orientations that are used in the perturbation models derived in Sec. 4 .

\section{Perturbation Models}

The estimated velocity components at the resonator spokes are given by Eq. (1) and are equal to those of a thin, inextensible ring when the nondominant $\theta$ harmonics are neglected. Thus, a perturbation model for the resonator can be developed by first determining a perturbation model for a thin ring subjected to point-mass perturbations. In general, a modal frequency, $\omega_{0}$, of a linear elastic structure is determined from the Rayleigh quotient $\omega_{0}^{2}=P_{0} / T_{0}$, in which $P_{0}$ is the maximum stored elastic energy and $\omega_{0}^{2} T_{0}$ is the maximum kinetic energy in the mode. If it is assumed that only the kinetic energy of the mode is perturbed when a perturbing mass is added to, or subtracted from, the structure, then an estimate of the perturbed modal frequency $\omega_{0}+\delta_{\omega}$ can be determined from the perturbed Rayleigh quotient, $\left(\omega_{0}+\delta_{\omega}\right)^{2}=P_{0} /\left(T_{0}+\delta_{T}\right)$, where $\omega_{0}^{2} \delta_{T}$ is the approximate change in kinetic energy due to the mass perturbation. The change in modal frequency can then be approximated as $\delta_{\omega} \approx-0.5 \omega_{0} \delta_{T} /$ $T_{0}$. This expression will be used below to relate the pre and postperturbation modal frequency difference within nominally degenerate pairs of modes in a thin ring.

A thin, uniform ring possesses pairs of degenerate modes. If the $n$th pair of modes is assumed to possess modal frequencies at $\omega_{0}$, then these modal frequencies decrease in frequency and typically 
detune when the ring is perturbed by adding a set of point masses. The perturbed modal frequencies can be estimated by computing the change in the kinetic energy of each mode, which is determined from knowledge of the mass locations in relation to the postperturbation mode shapes. Let the radial velocity component of a mode in the $n$th pair of modes be denoted $u_{1}$. The tangential component is denoted $w_{1}$. Similarly, $u_{2}$ and $w_{2}$ represent the radial and tangential velocity components associated with the companion mode. It is assumed that the first mode has modal frequency $\omega_{1}$ and the companion mode has modal frequency $\omega_{2}$. These velocity components are $u_{1}=U_{1}(\theta) e^{j \omega_{1} t}, w_{1}(t)=W_{1}(\theta) e^{j \omega_{1} t}$, $u_{2}=U_{2}(\theta) e^{j \omega_{2} t}$, and $w_{2}=W_{2}(\theta) e^{j \omega_{2} t}$, where the mode shapes are closely approximated by

$$
\begin{aligned}
& U_{1}(\theta)=\alpha \cos \left(n\left(\theta-\psi_{n, 1}\right)\right), \quad W_{1}(\theta)=\beta \sin \left(n\left(\theta-\psi_{n, 1}\right)\right) \\
& U_{2}(\theta)=\alpha \sin \left(n\left(\theta-\psi_{n, 1}\right)\right), \quad W_{2}(\theta)=-\beta \cos \left(n\left(\theta-\psi_{n, 1}\right)\right)
\end{aligned}
$$

The nominal kinetic energy $\omega_{0}^{2} T_{o}$ can be taken to be the same in each mode because the analysis given below only relies on $\alpha^{2} / T_{0}$, which is independent of the amplitude so, without loss of generality, the amplitudes $\alpha$ and $\beta$ are chosen to be the same for each mode. The angle $\psi_{n, 1}$, respectively, $\psi_{n, 2}$, is where the radial velocity achieves its largest value for the $\omega_{1}$ mode, respectively, $\omega_{2}$ mode-these are modes' radial antinodes. The mode shape expressions have used the fact that $\psi_{n, 2}=\psi_{n, 1} \pm \pi / 2 n$ radians, cf., the radial antinode measurements in Figs. 10 and 11.

Consider a mass of size $m_{0}$ that is the reference mass against which all other masses are scaled. The introduction of a reference mass is motivated by the fact that in the resonator under consideration, only solder spheres of a fixed diameter can be applied to the resonator-see Fig. 2. When a single $m_{0}$ is affixed at angle $\theta_{0}$ to a perfect ring, a radial antinode at $\psi_{n, 1}=\theta_{0}$ is created for the lower frequency mode which, without loss of generality, is identified with $\omega_{1}$. The degenerate modal frequencies $\omega_{0}$ are perturbed to $\omega_{1}$ and $\omega_{2}$ according to $\omega_{1}-\omega_{0}=\delta_{\omega}=-0.5 \omega_{0} \delta_{T} / T_{0}$, where $\delta_{T}=0.5 m_{0}\left(U_{1}^{2}\left(\theta_{0}\right)+W_{1}^{2}\left(\theta_{0}\right)\right)=0.5 m_{0} \alpha^{2}$, and similarly, $\omega_{2}-\omega_{0}=$ $\delta_{\omega}=-0.5 \omega_{0} \delta_{T} / T_{0}$, where $\delta_{T}=0.5 m_{0}\left(U_{2}^{2}\left(\theta_{0}\right)+W_{2}^{2}\left(\theta_{0}\right)\right)=0.5 m_{0} \beta^{2}$. The sensitivity, $\gamma_{n}$, associated with the $n$th pair of modes is defined to be the difference of the modal frequencies under this perturbation scenario, which, by the labeling convention, is positive

$$
\gamma_{n}=\omega_{2}-\omega_{1}=\frac{1}{4} \frac{\omega_{0}}{T_{0}}\left(\alpha^{2}-\beta^{2}\right) m_{0}
$$

The sensitivity is interpreted as the (positive) modal frequency difference that is observed when placing a reference mass on the perfect ring. It is experimentally measured in practice by placing a perturbing mass on the lower frequency mode radial antinode and observing the change in modal frequency difference. Note that the square of the amplitudes scale with $T_{0}$ so $\gamma_{n}$ is independent of the energy in the modes.

In order to develop an expression relating pre and postperturbation frequency differences, we consider the scenario in which $N$ point masses, denoted $m_{p}, p=1, \ldots, N$, are applied to an initially uniform thin ring at points $\theta_{p}$. The radial antinode orientation $\psi_{n, 1}$ associated with the lower frequency mode, $\omega_{1}$, was derived in Ref. [14] to be $\tan 2 n \psi_{n, 1}=\sum_{p} m_{p} \sin 2 n \theta_{p} / \sum_{p} m_{p} \cos 2 n \theta_{p}$, assuming the point masses are sufficiently small. The difference between the perturbed modal frequencies in the $n$th pair of modes is defined $\Delta_{n}=\omega_{2}-\omega_{1}$

$$
\begin{aligned}
\Delta_{n} & =-\frac{1}{2} \frac{\omega_{0}}{T_{0}} \sum_{p=1}^{N} m_{p}\left(U_{2}^{2}\left(\theta_{p}\right)+W_{2}^{2}\left(\theta_{p}\right)-U_{1}^{2}\left(\theta_{p}\right)-W_{1}^{2}\left(\theta_{p}\right)\right) \\
& =\frac{\gamma_{n}}{m_{0}} \sum_{p=1}^{N} m_{p} \cos \left(2 n\left(\theta_{p}-\psi_{n, 1}\right)\right)
\end{aligned}
$$

Thus, if a perfect ring is perturbed by point mass, the effect of the masses can be aggregated into a single "equivalent" mass $m_{e}=\sum_{p} m_{p} \cos \left(2 n\left(\theta_{p}-\psi_{n, 1}\right)\right)$ located at $\psi_{n, 1}$. In practice, the equivalent mass is calculated from the sensitivity, reference mass value, and measured frequency split, i.e., $m_{e}=m_{0} \Delta_{n} / \gamma_{n}$. Note that

$$
m_{e}=\cos 2 n \psi_{n, 1} \sum_{p=1}^{N} m_{p} \cos 2 n \theta_{p}+\sin 2 n \psi_{n, 1} \sum_{p=1}^{N} m_{p} \sin 2 n \theta_{p}
$$

from which it follows:

$$
\begin{aligned}
& m_{e} \cos 2 n \psi_{n, 1}=\sum_{p} m_{p} \cos 2 n \theta_{p} \\
& m_{e} \sin 2 n \psi_{n, 1}=\sum_{p} m_{p} \sin 2 n \theta_{p} .
\end{aligned}
$$

Thus, the equivalent mass can be used in place of the $N$ initial masses in all subsequent computations.

We now take the perspective that the ring with $N$ masses is the initial state of the resonator. The initial state may be a consequence of fabrication imperfections, masses deposited in previous tuning steps, or a combination of the two. The only parameters we require from the resonator in this initial state are the modal frequency difference, $\Delta_{n}$, and low-frequency mode antinode orientation, $\psi_{n, 1}$. These parameters are readily obtained using the measurement and modeling technique discussed in Sec. 3. In any case, we consider adding a reference mass $m_{0}$ at $\theta_{0}$. This new mass is a perturbation to the resonator's initial state so it is of interest to develop an expression relating the preperturbation frequency difference, $\Delta_{n}$, and antinode orientation, $\psi_{n, 1}$, to the postperturbation frequency difference, $\tilde{\Delta}_{n}$, and antinode orientation, $\psi_{n, 1}$. The postperturbation antinode is given by

$$
\begin{aligned}
\tan 2 n \tilde{\psi}_{n, 1}= & \frac{m_{0} \sin 2 n \theta_{0}+\sum_{p} m_{p} \sin 2 n \theta_{p}}{m_{0} \cos 2 n \theta_{0}+\sum_{p} m_{p} \cos 2 n \theta_{p}} \\
& =\frac{m_{0} \sin 2 n \theta_{0}+m_{e} \sin 2 n \psi_{n, 1}}{m_{0} \cos 2 n \theta_{0}+m_{e} \cos 2 n \psi_{n, 1}} .
\end{aligned}
$$

The postperturbation frequency difference is

$$
\tilde{\Delta}_{n}=\gamma_{n}\left(\cos \left(2 n\left(\theta_{0}-\tilde{\psi}_{n, 1}\right)\right)+\frac{1}{m_{0}} \sum_{p=1}^{N} m_{p} \cos \left(2 n\left(\theta_{p}-\tilde{\psi}_{n, 1}\right)\right)\right)
$$

which simplifies to

$$
\begin{aligned}
\tilde{\Delta}_{n}= & \left(\gamma_{n} \cos 2 n \theta_{0}+\Delta_{n} \cos 2 n \psi_{n, 1}\right) \sin 2 n \tilde{\psi}_{n, 1} \\
& +\left(\gamma_{n} \sin 2 n \theta_{0}+\Delta_{n} \sin 2 n \psi_{n, 1}\right) \sin 2 n \tilde{\psi}_{n, 1}
\end{aligned}
$$

In view of Eq. (3)

$$
\begin{aligned}
& \tilde{\Delta}_{n} \cos 2 n \tilde{\psi}_{n, 1}=\Delta_{n} \cos 2 n \psi_{n, 1}+\gamma_{n} \cos 2 n \theta_{0} \\
& \tilde{\Delta}_{n} \sin 2 n \tilde{\psi}_{n, 1}=\Delta_{n} \sin 2 n \psi_{n, 1}+\gamma_{n} \sin 2 n \theta_{0}
\end{aligned}
$$

which can be compactly expressed as

$$
\tilde{\Delta}_{n} e^{j 2 n \tilde{\psi}_{n, 1}}=\Delta_{n} e^{j 2 n \psi_{n, 1}}+\gamma_{n} e^{j 2 n \theta_{0}}
$$

where $j=\sqrt{-1}$.

Extending Eq. (4) to the resonator under study is straightforward. First, it is assumed that reference masses can be placed on any one of the 96 large spokes (if a spoke is unavailable, it can be excluded from the search procedure described in Sec. 5). Targeting more than one spoke just extends the term with $\gamma_{n}$ in Eq. (4) to a sum; however, a correction must be made to the velocity component amplitudes because they vary among spoke layers. The spoke 
layer index, $k$, is used to adjust the $\alpha$ and $\beta$ according to Table 1 , and definition of $\gamma_{n}$ is modified to account for using a single sensitivity across all spoke layers

$$
\tilde{\Delta}_{n} e^{j 2 n \tilde{\psi}_{n, 1}}=\Delta_{n} e^{j 2 n \psi_{n, 1}}+\gamma_{n} \sum_{k, i} \mu_{k, i}\left(\alpha_{n, k}^{2}-\beta_{n, k}^{2}\right) e^{j 2 n \theta_{k, i}}
$$

Recall that the indices $(k, i)$ are used to uniquely identify a spoke (Fig. 1). If spoke $(k, i)$ receives a solder sphere of mass $m_{0}$, then $\mu_{k, i}=1$; otherwise, $\mu_{k, i}=0$. This is a useful representation for the optimization formulated in Sec. 5 to determine spoke locations for which $\Delta_{2}$ and $\Delta_{3}$ are simultaneously reduced. As in the case of the ring, the sensitivity $\gamma_{n}$ is related to the change in the frequency split if the reference mass $m_{0}$ is placed on the antinode of the innermost spoke layer $(k=1)$. If it is placed on the lower frequency mode's antinode, then the frequency split is increased by $\gamma_{n}\left(1-\beta_{n, 1}^{2}\right)$. Likewise, if the reference mass is placed on the higher frequency mode's antinode, then the pre and postdeposition frequency splits are reduced by $\gamma_{n}\left(1-\beta_{n, 1}^{2}\right)$ since $\alpha_{n, 1}=1$. If mass is deposited at an antinode on other spoke layers $(k=2,3,4)$, then the change in frequency split is $\gamma_{n}\left(\alpha_{n, k}^{2}-\beta_{n, k}^{2}\right)$.

\section{Algorithm for Selection of Deposition Sites}

Reducing the modal frequency difference within the $n=2$ pair of modes has been demonstrated in Refs. [7] and [8] for the resonator under consideration. In the case of mass loading, the general strategy is to deposit mass in a neighborhood of the antinodes of the higher frequency mode since this has a greater effect on modifying the kinetic energy of the higher frequency mode relative to the lower frequency mode. When mass is removed, the antinodes of the lower frequency mode are targeted. In either case, a search over the spoke locations in a neighborhood of the relevant antinodes is sufficient to produce tuned modes after several iterations. This process ignores the effects of mass loading on other modes, though. When the objective is to simultaneously reduce $\Delta_{2}$ and $\Delta_{3}$, this heuristic approach is of limited utility because the $n=2$ and $n=3$ antinodes are not aligned in any way. Adding the quantization constraints on the permissible mass and deposition locations further complicates the search for deposition locations that yield a satisfactory reduction in frequency split. An exhaustive search is prohibitive because typically 10-20 spokes are modified out of 96 possible locations. Thus, an alternative search procedure is necessary. The expression relating the pre and postperturbation frequency splits (5) can be incorporated into a mixed-integer linear programming problem [18] for identifying promising mass deposition locations in order to simultaneously reduce both the $n=2$ and $n=3$ postperturbation frequency splits relative to their preperturbation values. The optimization problem is stated as follows:

$$
\begin{aligned}
& \text { minimize } \zeta \\
& \text { subject to: } \\
& \qquad \in \mathbf{R}, \zeta>0 \\
& \mu_{k, i} \in\{0,1\} \\
& -\zeta \leqslant \tilde{\Delta}_{2} \cos \left(4 \tilde{\psi}_{2,1}\right) \leqslant \zeta \\
& -\zeta \leqslant \tilde{\Delta}_{2} \sin \left(4 \tilde{\psi}_{2,1}\right) \leqslant \zeta \\
& -\zeta \leqslant \tilde{\Delta}_{3} \cos \left(6 \tilde{\psi}_{3,1}\right) \leqslant \zeta \\
& -\zeta \leqslant \tilde{\Delta}_{3} \sin \left(6 \tilde{\psi}_{3,1}\right) \leqslant \zeta \\
& \sum_{k, i} \mu_{k, i} \leqslant c
\end{aligned}
$$

Here, $c$ is a positive integer that sets a bound on the total number of masses that $\underset{\sim}{a}$ permitted. Note that $\tilde{\Delta}_{2} \cos \left(4 \tilde{\psi}_{2,1}\right), \tilde{\Delta}_{2} \sin$ $\left(4 \tilde{\psi}_{2,1}\right), \tilde{\Delta}_{3} \cos \left(6 \tilde{\psi}_{3,1}\right)$, and $\tilde{\Delta}_{3} \sin \left(6 \tilde{\psi}_{3,1}\right)$ are affine functions of the decision variables $\mu_{k, i}$ per Eq. (5). Despite the difficulty of finding a global minimum, there exist widely available computational tools to estimate the minimum. Furthermore, we are not overly concerned with finding the deposition pattern(s) that yield the minimal value of $\zeta$ because the expressions for $\tilde{\Delta}_{2}$ and $\tilde{\Delta}_{3}$ are approximate anyway. Thus, it is more prudent to set the maximum number of solder spheres to be deposited and then terminate the search if $\zeta$ is reduced to a sufficient degree. The gradual reduction of $\Delta_{2}$ and $\Delta_{3}$ to the desired level is then achieved over several iterations of measurement-computation-deposition. Note that for a given deposition pattern that satisfies the constraints in Eq. (6), the predicted frequency differences will satisfy $\left|\tilde{\Delta}_{2}\right| \leq \sqrt{2} \zeta$ and $\left|\tilde{\Delta}_{2}\right| \leq \sqrt{2} \zeta$.

\section{Demonstration of Proposed Tuning Technique}

Simultaneous tuning of the $n=2,3$ pairs of modes is demonstrated for the resonator whose frequency responses are shown in Figs. 3, 7, and 8. The initial frequency splits are $23.49 \mathrm{~Hz}$ and $2.43 \mathrm{~Hz}$ for the $n=2$ pair and $n=3$ pair, respectively. The first task is to find the sensitivity parameters $\gamma_{n}$ for $n=2,3$. This is accomplished by placing mass perturbations on the resonator and comparing the frequency splits and antinode orientations before and after the deposition using Eq. (5). These sensitivities are established using resonators other than one under consideration and can be considered fixed for a given resonator design, i.e., they do not need to be determined for each device. Two sizes of solder spheres are used for mass perturbations in this study. The larger solder spheres average $75 \mu \mathrm{m}$ in diameter and the small solder spheres average $35 \mu \mathrm{m}$ in diameter. The estimated average sensitivity values for the $75 \mu \mathrm{m}$ diameter solder spheres are $\gamma_{2}=5.0 \mathrm{~Hz}$ and $\gamma_{3}=4.5 \mathrm{~Hz}$. These values are scaled by 0.1 for the $35 \mu \mathrm{m}$ diameter solder spheres.

The optimization (6) is used to determine the deposition sites. After the initial modal measurements are performed, locations for nine large solder spheres were determined. The optimization requires about one minute to determine the deposition sites. MATLAB software was used for these computations [19]. The sites selected by the algorithm are shown in Fig. 12 and Table 2

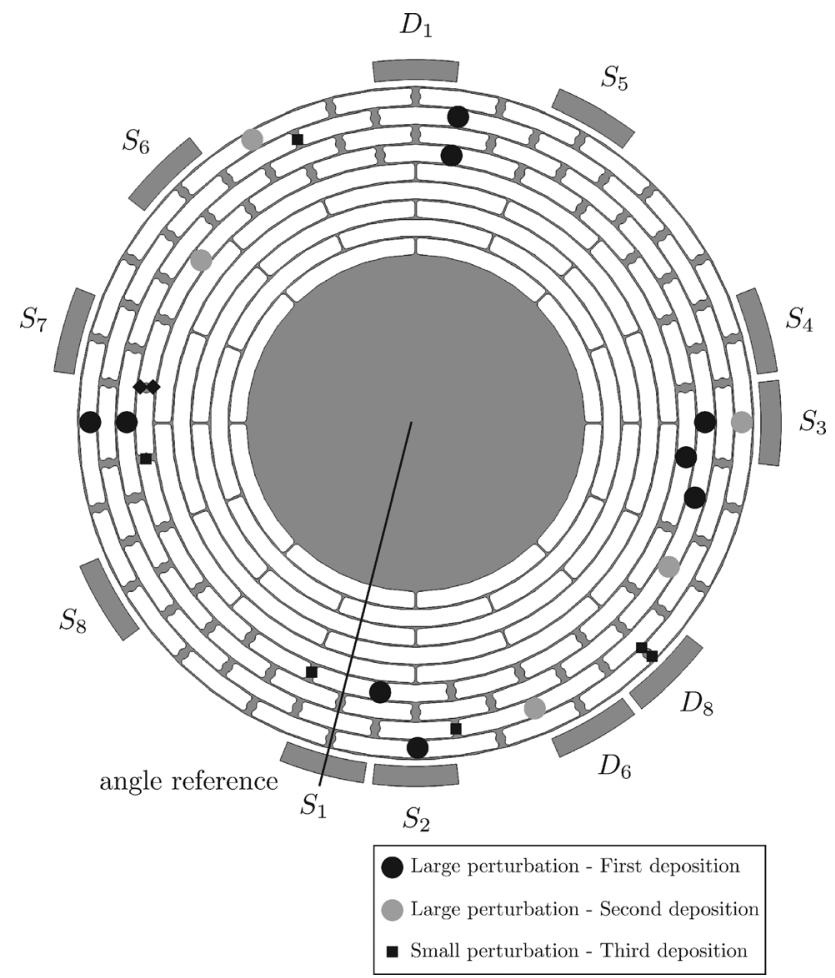

Fig. 12 The map of masses placed on the resonator in the deposition steps 
Table 2 Summary of deposition steps

\begin{tabular}{|c|c|c|c|c|c|}
\hline Iteration & $\Delta_{2}(\mathrm{~Hz})$ & $\Delta_{3}(\mathrm{~Hz})$ & No. solder spheres & $Q_{2}(\mathrm{k})$ & $Q_{3}(\mathrm{k})$ \\
\hline Initial & 23.49 & 2.43 & - & $45.4,45.5$ & $32.1,32.3$ \\
\hline \multicolumn{6}{|c|}{ First deposition } \\
\hline Measured & 3.67 & 1.06 & $9 \mathrm{~L}^{\mathrm{a}}$ & $44.4,44.4$ & $31.7,31.8$ \\
\hline Predicted & 3.34 & 0.49 & - & - & - \\
\hline \multicolumn{6}{|c|}{ Second deposition } \\
\hline Measured & 0.72 & 1.17 & $5 \mathrm{~L}$ & $44.4,44.5$ & $31.8,31.5$ \\
\hline Predicted & 0.14 & 0.20 & & - & - \\
\hline \multicolumn{6}{|c|}{ Third deposition } \\
\hline Measured & 0.11 & 0.16 & $8 \mathrm{~S}$ & $44.2,44.2$ & $31.3,31.5$ \\
\hline Predicted & 0.04 & 0.16 & & - & - \\
\hline
\end{tabular}

a" $L$ " denotes $75 \mu \mathrm{m}$ diameter solder sphere, "S" denotes $35 \mu \mathrm{m}$ diameter solder sphere.

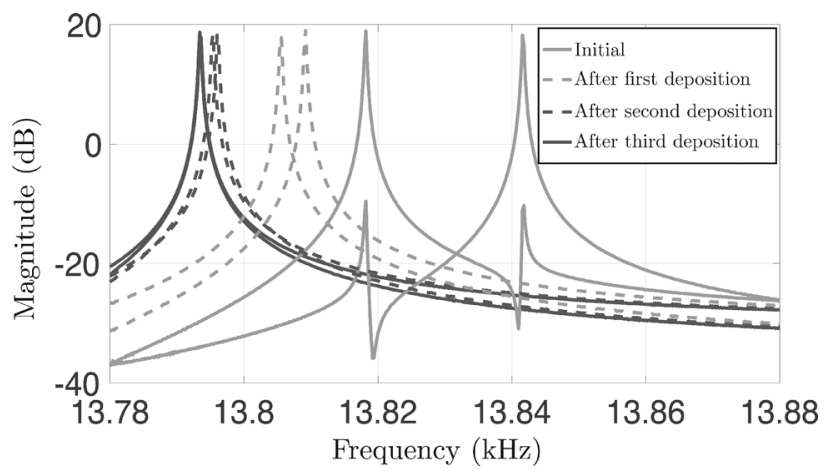

Fig. 13 Empirical frequency response of the $n=2$ pair of modes for the $S_{2} / D_{1}$ and $S_{6} / D_{8}$ measurement channels. The initial modal frequency difference of $23.49 \mathrm{~Hz}$ is reduced to $0.11 \mathrm{~Hz}$ after three rounds of mass deposition.

summarizes the $n=2,3$ frequency differences at each step of the process. In the first iteration, the $n=2$ frequency difference was substantially reduced while the $n=3$ was reduced by a factor of 2.3. The modal parameters were measured once again and sites were selected for five large solder spheres. The model predicts a further reduction in the frequency differences within each pair; however, the measurements indicate that $n=3$ difference actually slightly increases. Despite this, the next iteration using the smaller solder spheres reduces the differences to less than $200 \mathrm{mHz}$ within each pair of modes. The empirical frequency responses in a neighborhood of the $n=2$ and $n=3$ pairs of modes are shown in Figs. 13 and 14.

Table 2 also reports the quality factors $(Q)$ of each mode. It appears that the quality factors are not impacted by this tuning procedure. This was also noted in Ref. [7] for the $n=2$ pair. One point of note is the fact that more deposition is required for the results reported here compared to the results in Ref. [7], which exclusively focused on manipulating the $n=2$ modes. For example, less than ten $75 \mu \mathrm{m}$ solder spheres were required to eliminate about the same initial $n=2$ modal frequency difference in Ref. [7]; however, approximately 16 solder spheres of the same size were necessary for the simultaneous manipulation of the $n=2,3$ modal frequencies. This is not surprising because the additional constraint of tuning the $n=3$ pair precludes the most efficient solution for the $n=2$ pair.

The postperturbation frequency differences are predicted reasonably well by the model after the first deposition, however, there is considerable error between the measured versus predicted $\Delta_{3}$ after the second deposition. The source(s) of the error have not been definitively identified. One source of uncertainty is the actual quantity of mass that is deposited. The diameters of the spheres are not measured prior to deposition so any variability in the diameters of the solder spheres will change the deposited mass

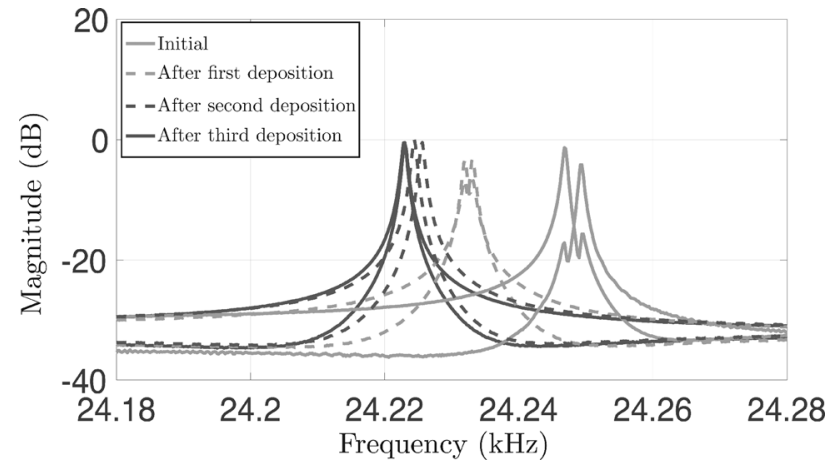

Fig. 14 Empirical frequency response of the $n=3$ for the $S_{2} / D_{1}$ and $S_{5} / D_{6}$ measurement channels. The initial modal frequency difference of $2.43 \mathrm{~Hz}$ is reduced to $0.16 \mathrm{~Hz}$ after three rounds of deposition.

from the assumed reference mass $m_{0}$. This uncertainty can be mitigated by pursuing the mass removal technique introduced in Ref. [8] since the etch rates are well documented. Furthermore, postetch measurements can be performed to very accurately determine the amount of material removed.

Other approximations in the model relate to the spoke velocities. After the $2 \theta$ "fundamental," the most significant harmonic component in the mode shapes of the $n=2$ modes is the $6 \theta$ term; however, the magnitude of this term is about $1 \%$ of the magnitude of the $2 \theta$ harmonic for the outer ring radial displacement. According to Fig. 5, though, the amplitude of the $6 \theta$ harmonic in the radial motion of the inner ring adjacent to the innermost layer of large spokes $(k=1$ in Fig. 1$)$ is about $5 \%$ of the outermost ring radial displacement amplitude-in this case, it may be possible to detect the $6 \theta$ harmonic. Although it is difficult to quantify the net impact of the neglected harmonics and mass uncertainty, it seems unlikely to produce the error noted in $\Delta_{3}$ at the second deposition step. Thus, we should reflect on potential deficiencies in Eq. (4) for the perturbed ring from which Eq. (5) was derived. It is possible to challenge the assumption that pure point mass perturbations only affect the kinetic energy of the mode. In a resonator in which the mode of interest is isolated, the mode shape is not expected to change much under small perturbations. Thus, if small masses are added to the resonator, the strain energy remains essentially the same because the strain energy integral is performed over the same mode shape, so constraining changes to only occur in the kinetic energy is reasonable. In a resonator in which modes are degenerate, however, the mode shape orientation is sensitive to the perturbations despite the fact that the shape, i.e., elliptical, for the $n=2$ modes in a ring resonator, is relatively robust to the perturbations. The change in orientation, however, can modify the strain energy in this scenario, because, in reality, local variations in beam widths, spoke sizes, and so forth, affect both the mass and stiffness distribution of the resonator. These variations are difficult, if not impossible, to directly measure: only their aggregate impact on the modal properties is measurable. The results in Refs. [7] and [8] did not appear to suffer from large prediction errors; however, because the point mass perturbations were largely confined to a neighborhood of antinodes, the postperturbation antinode orientations tended to remain close to their preperturbation orientations. In this case, the strain energy would remain essentially the same for a given mode. This requires further investigation, but despite any deficiencies of the model, the iterative nature of the tuning process appears to be somewhat self-correcting and so the challenge of tuning both $n=2$ and $n=3$ pairs of modes proved feasible in three iterations.

\section{Conclusion}

A systematic postfabrication technique is proposed for the simultaneous reduction of the modal frequency differences 
between the $n=2$ and $n=3$ modes in an axisymmetric resonator. The technique is demonstrated on a resonator in which the initial modal frequency differences were $23.5 \mathrm{~Hz}$ and $2.4 \mathrm{~Hz}$ for the $n=2$ and $n=3$ pairs, respectively. After three iterations, the modal frequency differences were reduced to less than $200 \mathrm{mHz}$. The physical modification of the resonator is achieved by mass loading specific areas on the resonator with solder spheres of two different diameters. An approximate model for relating the pre and postdeposition modal frequency differences within each pair was incorporated into a search procedure to select a prespecified number of deposition locations. The algorithm requires the initial frequency difference within each pair as well as information on the mode shape orientations. This information is obtained by analyzing models fit to the resonator transient response. The statespace models are developed using data from eight pick-off locations situated around the outermost ring of the resonator. The large number of pick-off allows the robust identification of the mode shape orientations even with sub-Hertz modal frequency differences.

\section{Acknowledgment}

The authors thank Howie Ge, Tom Lee, and the UCLA Nanolab staff for their assistance.

\section{References}

[1] Shcheglov, K. V., and Challoner, A. D., 2006, "Isolated Planar Gyroscope With Internal Radial Sensing and Actuation," U.S. Patent No. 7040163B2.

[2] Nitzan, S., Ahn, C., Su, T.-H., Li, M., Ng, E., Wang, S., Yang, Z., O’Brien, G., Boser, B., Kenny, T., and Horsley, D., 2013, "Epitaxially-Encapsulated Polysilicon Disk Resonator Gyroscope," IEEE MEMS, Taipei, Taiwan, Jan. 20-24, pp. 625-628.

[3] Kim, D., and M'Closkey, R., 2014, "A MEM Vibratory Gyro With ModeMatching Achieved by Resonator Mass Loading," IEEE/ION Position, Location and Navigation Symposium, Monterey, CA, May 5-8, pp. 499-503.
[4] Challoner, A., Ge, H., and Liu, J., 2014, "Boeing Disc Resonator Gyroscope," IEEE/ION Position, Location and Navigation Symposium, Monterey, CA, May 5-8, pp. 504-514.

[5] Kim, D., and M'Closkey, R. T., 2013, "Spectral Analysis of Vibratory Gyro Noise," IEEE Sens. J., 13(11), pp. 4361-4374.

[6] Ge, H., and M'Closkey, R., 2017, "Simultaneous Angular Rate Estimates Extracted From a Single Axisymmetric Resonator,” IEEE Sens. J., 17(22), pp. 7460-7469.

[7] Schwartz, D. M., Kim, D., Stupar, P., DeNatale, J., and M'Closkey, R. T., 2015, "Modal Parameter Tuning of an Axisymmetric Resonator Via Mass Perturbation," J. Microelectromech. Syst., 24(3), pp. 545-555.

8] Behbahani, A. H., Kim, D., Stupar, P., DeNatale, J., and M'Closkey, R. T., 2017, "Tailored Etch Profiles for Wafer-Level Frequency Tuning of Axisymmetric Resonators," J. Microelectromech. Syst., 26(2), pp. 333-343.

[9] Bernstein, J., Bancu, M., Cook, E., Henry, T., Kwok, P., Nyinjee, T., Perlin, G., Teynor, W., and Weinberg, M., 2014, "Diamond Hemispherical Resonator Fabrication by Isotropic Glass Etch," Solid-State Sensors, Actuators and Microsystems Workshop, Hilton Head, SC, June 8-12, pp. 273-276.

[10] Gallacher, B., Hedley, J., Burdess, J., Harris, A., and McNie, M., 2003, "Multimodal Tuning of a Vibrating Ring Using Laser Ablation," Proc. Inst. Mech. Eng., Part C, 217(5), pp. 557-576.

[11] Rourke, A., McWilliam, S., and Fox, C., 2002, "Multi-Mode Trimming of Imperfect Thin Rings Using Masses at Pre-Selected Locations," J. Sound Vib. 256(2), pp. 319-345.

[12] Bisegna, P., and Caruso, G., 2007, "Frequency Split and Vibration Localization in Imperfect Rings," J. Sound Vib., 306(3-5), pp. 691-711.

[13] Choi, S.-Y., and Kim, J.-H., 2011, "Natural Frequency Split Estimation for Inextensional Vibration of Imperfect Hemispherical Shell," J. Sound Vib. 330(9), pp. 2094-2106.

[14] Fox, C., 1990, "A Simple Theory for the Analysis and Correction of Frequency Splitting in Slightly Imperfect Rings,” J. Sound Vib., 142(2), pp. 227-243.

[15] Rourke, A. K., McWilliam, S., and Fox, C. H. J., 2001, "Multi-Mode Trimming of Imperfect Rings," J. Sound Vib., 248(4), pp. 695-724.

[16] Rao, S. S., 2007, Vibration of Continuous Systems, Wiley, Hoboken, NJ.

[17] Lorentz, T., Kim, D., and M'Closkey, R. T., 2014, "A Novel Technique for Extracting Parametric Models From Mem Resonator Test Data," International Symposium on Inertial Sensors and Systems (ISISS), Laguna Beach, CA, Feb. 25-26, pp. 1-4.

[18] Bradley, S. P., Hax, A. C., and Magnanti, T. L., 1977, Applied Mathematical Programming, Addison-Wesley, Boston, MA.

[19] The Mathworks, Inc., 2017, "MATLAB R2017b," The Mathworks, Natick, MA. 